\title{
Design, synthesis, docking, Hirshfeld surface analysis and DFT calculations of 2-methylxanthen-9- with the FtsZ protein from Staphylococcus aureus
}

\author{
V Lakshmi Ranganatha1 ${ }^{1}$, Mallikarjunaswamy $C^{2}$, Jagadeep Chandra ${ }^{3}$, Ramith Ramu4 ${ }^{\$}$, Prithvi S \\ Shirahatti 5, Naveen Kumar6, Sowmya B P6, Hussien Ahmed Khamees7, Mahendra \\ Madegowda7 , Shaukath Ara Khanum ${ }^{8 *}$
}

1Department of Chemistry, The National Institute of Engineering, Manandavadi Road, Mysuru 570008, Karnataka, India; 2 PG Department of Chemistry, JSS College of Arts, Commerce and Science, Ooty Road, Mysuru-570025, Karnataka, India; ${ }^{3}$ Department of Microbiology, School of Life Sciences, JSS Academy of Higher Education and Research, SS Nagar, Mysuru-570015, karnataka, India; ${ }^{4}$ Department of Biotechnology and Bioinformatics, School of Life Sciences, JSS Academy of Higher Education and Research, SS Nagar, Mysuru-570015, Karnataka, India; ${ }^{5}$ Department of Biotechnology, Teresian College, Siddhartha Nagara, Mysuru-570011, karnataka, India; ${ }^{6}$ Department of Chemistry, Sri Dharmasthala Manjunatheshwara College, Ujire - 574240, karnataka, India; ${ }^{7}$ Department of Studies in Physics, Manasagangotri, University of Mysore, Mysuru-570006, Karnataka, India; ${ }^{8}$ Department of Chemistry, Yuvaraja's College (Autonomous), University of Mysore, Mysuru-570 005, karnataka, India; *Correspondence Dr. Shaukath Ara Khanum Phone: +91 - 99018 88755, Fax: +821- 2419239 - Email: shaukathara@yahoo.co.in; \$Co-correspondence Dr. Ramith Ramu - Phone: +91 9986380920, Email: ramith24prince@gmail.com

Received February 28, 2021; Revised March 10, 2021; Accepted March 11, 2021, Published March 31, 2021

DOI: $10.6026 / 97320630017393$

Declaration on official E-mail:

The corresponding author declares that official e-mail from their institution is not available for all authors

Declaration on Publication Ethics:

The author's state that they adhere with COPE guidelines on publishing ethics as described elsewhere at https:// publicationethics.org/. The authors also undertake that they are not associated with any other third party (governmental or non-governmental agencies) linking with any form of unethical issues connecting to this publication. The authors also declare that they are not withholding any information that is misleading to the publisher in regard to this article.

\section{Abstract: function data in this context. \\ Keywords: Synthesis, 2-Methylxanthen-9-one, antimicrobial, docking and DFT.}

It is of interest to document the design, synthesis, docking, Hirshfeld surface analysis and DFT calculations of 2-methylxanthen-9-with the FtsZ protein (PDB ID: 3VOB) from Staphylococcus aureus for antimicrobial applications. We report the quantitative structure

\section{Background:}

Xanthones are natural or synthetic compounds which are structurally related to anthraquinones and among these mitoxantrone is a well-established anti-cancer drug. [1,2] Extracts of the pericarp of ripe fruits possess immuno modulating [3], antibacterial [4], anti-mutagenic [5,6], anti-cancer [7] and other pharmacological activities. It is known that naturally occurring pyranoxanthones are more active than dihydropyrano xanthones in terms of their biological activities [8]. Xanthones also concern semi-synthetic and synthetic heterocyclic compounds with the dibenzo- $\gamma$-pyrone scaffold. Interest on xanthone analogues has been growing considerably due to the wide range of pharmacological applications exhibited by this group of compounds, including anticancer, immuno modulation and other promising activities [9-13]. Therefore, it is of interest to document the design, synthesis, docking, Hirshfeld surface analysis and DFT calculations of 2-methylxanthen-9-with the FtsZ protein 
(PDB ID: 3VOB) from Staphylococcus aureus for antimicrobial applications.

\section{Materials and Methods:}

All solvents and reagents were purchased from Sigma Aldrich Chemicals Pvt Ltd., USA. Melting points were determined on an electrically heated VMP-III melting point apparatus. The FT-IR spectra were recorded using $\mathrm{KBr}$ discs and Nujol on FT-IR Jasco 4100 infrared spectrophotometer. ${ }^{1} \mathrm{H}$ NMR spectra were recorded using Bruker DRX 400 spectrometer at $400 \mathrm{MHz}$ with TMS as an internal standard. Mass spectra were recorded on LC-MS/MS (API-4000) mass spectrometer. Further, the elemental analysis of the compounds was performed on a Perkin Elmer 2400 elemental analyzer. The synthesis of proposed compound 2-methylxanthen9 -one was outlined in the scheme 1 . The starting material phenyl benzoates (3) were prepared according to a reported procedure through the reaction of $p$-cresol (1) with benzoyl chloride (2) in the presence of $10 \%$ sodium hydroxide as a base. Compound 3 on subjected to Fries rearrangement afforded substituted diaryl methanone commonly known as hydroxy benzophenone (4). Compound 4 on reaction with ethyl chloroacetate resulted in ethyl [2-benzoyl-4-methylphenoxy] acetate (5) in excellent yield. Further, Compounds 5 with $10 \% \mathrm{NaOH}$ in the presence of ethyl alcohol after refluxing gave 2-methylxanthen-9-one (6).

\section{Preparation of 4-methyl phenyl benzoate:}

4-Methyl phenyl benzoate (3) was synthesized by benzoylation of 4-methyl phenol (1) with benzoyl chloride $(\mathbf{2}, 1: 1)$ using $10 \%$ sodium hydroxide solution. The reaction mass was stirred for about 2-3 hours at $0^{\circ} \mathrm{C}$. The reaction was monitored by TLC using 4:1 n-hexane: ethyl acetate as a mobile phase. After completion of the reaction the oily product was extracted with ether layer. Ether layer was washed with $10 \%$ sodium hydroxide solution $(3 \times 50 \mathrm{ml})$ followed by water $(3 \times 30 \mathrm{ml})$ and then dried over anhydrous sodium sulphate and evaporated the solvent under pressure to afford compound 3 [14]. A pale-yellow liquid with $90 \%$ yield was obtained. IR (Neat): $1715 \mathrm{~cm}^{-1}(\mathrm{C}=\mathrm{O}) .{ }^{1} \mathrm{H}$ NMR (DMSO): $\delta 2.45$ (s, 3H, Ar- $\left.\mathrm{CH}_{3}\right), 7.5-8.2$ (m, 9H, Ar-H). MS: $\mathrm{m} / \mathrm{z} 213(\mathrm{M}+1)$. Anal. Calcd. for $\mathrm{C}_{14} \mathrm{H}_{12} \mathrm{O}_{2}$ (212): C, 79.22; $\mathrm{H}, 5.70$. Found: C, 79.18; H, $5.76 \%$.

Preparation of (2-Hydroxy-5-methylphenyl) phenyl methanone: (2-Hydroxy-5-methylphenyl) phenyl methanone commonly known as hydroxy benzophenone (4) was synthesized by Fries rearrangement. Compound $3(0.001 \mathrm{~mol})$ was treated with anhydrous aluminum chloride $(0.002 \mathrm{~mol})$ as a catalyst at $150-$ $170^{\circ} \mathrm{C}$ temperature without the presence of solvent for about 23 hours. The reaction mixture was then cooled to room temperature and quenched with $6 \mathrm{~N} \mathrm{HCl}$ in the presence of ice water. The reaction mixture was stirred for about 2-3 hours, filtered to separate solids and re-crystallized with methanol to obtain (2-hydroxy-5-methylphenyl) phenyl methanone (4). Yield $85 \%, \mathrm{mp} 81-83{ }^{\circ} \mathrm{C}$; IR (Nujol): $1670(\mathrm{C}=\mathrm{O}), 3545-3649 \mathrm{~cm}^{-1}(\mathrm{OH}) ; 1 \mathrm{H}$ NMR $\left(\mathrm{CDCl}_{3}\right)$ d: 2.3 (s, 3H, $\left.\mathrm{CH}_{3}\right), 6.85-7.75(\mathrm{~m}, 8 \mathrm{H}, \mathrm{Ar}-\mathrm{H}), 12.05$ (br s, 1H, OH); MS: m/z $212(\mathrm{M}+1)$. Anal. Calcd. for $\mathrm{C}_{14} \mathrm{H}_{12} \mathrm{O}_{2}$ (212): C, 79.24; H, 5.66. Found: C, 79.26; H, 5.64\%.
Procedure for the Preparation of ethyl [2-benzoyl-4methylphenoxy] acetate:

Compound 5 was obtained by refluxing a mixture of compound 4 $(0.013 \mathrm{~mol})$ and ethyl chloroacetate $(0.026 \mathrm{~mol})$ in dry acetone $(50$ $\mathrm{ml})$ and anhydrous potassium carbonate $(0.019 \mathrm{~mol})$ for $7-8$ hours. The reaction mixture was cooled and solvent was removed by distillation. The residual mass was triturated with cold water to remove potassium carbonate and extracted with ether $(3 \times 50$ $\mathrm{ml})$. The ether layer was washed with $10 \%$ sodium hydroxide solution $(3 \times 50 \mathrm{ml})$ followed by water $(3 \times 30 \mathrm{ml})$ and then dried over anhydrous sodium sulphate and evaporated to dryness to obtain crude solid, which on recrystallization with ethanol afforded ethyl [2-benzoyl-4-methylphenoxy] acetate (5) [15]. Yield, 79\%; mp 61-63 OC; IR (Nujol): 1664 (C=O), $1760 \mathrm{~cm}^{-1}$ (ester $\mathrm{C}=\mathrm{O}) ;{ }^{1} \mathrm{H}$ NMR $\left(\mathrm{CDCl}_{3}\right): \mathrm{d} 1.2\left(\mathrm{t}, \mathrm{J}=7 \mathrm{~Hz}, 3 \mathrm{H}, \mathrm{CH}_{3}\right.$ of ester), $2.3(\mathrm{~s}$, $\left.3 \mathrm{H}, \mathrm{CH}_{3}\right), 4.1\left(\mathrm{q}, \mathrm{J}=6 \mathrm{~Hz}, 2 \mathrm{H}, \mathrm{CH}_{2}\right.$ of ester), $4.5\left(\mathrm{~s}, 2 \mathrm{H}, \mathrm{OCH}_{2}\right)$, 7.1-7.7 (m, 8H, Ar-H); MS: m/z $298(\mathrm{M}+1)$. Anal. Calcd. For $\mathrm{C}_{18} \mathrm{H}_{18} \mathrm{O}_{4}$ (298): C, 72.48; H, 6.04. Found: $\mathrm{C}, 72.46 ; \mathrm{H}, 6.02 \%$.

Preparation of 2-methylxanthen-9-one:

2-Methylxanthen-9-one (6) was obtained by the reaction of ethyl [2-benzoyl-4-methylphenoxy] acetate (5), in the presence of $\mathrm{NaOH}$ as a base and ethyl alcohol as a solvent $(50 \mathrm{ml})$ refluxed for about 7-8 hours and then cooled. The reaction mixture was neutralized, solvent removed by distillation. The residual mass was washed with water and recrystallized by methanol, gave 2methylxanthen-9-one (6). Yield 70\%, m.p. 369-373 K; IR (Nujol):1665 cm-1 (C=O); ${ }^{1} \mathrm{H}$ NMR (CDCl3): $\delta 2.3$ (s, 3H, Ar- $\left.\mathrm{CH}_{3}\right)$, 6.9-7.6 (bm, 7H, Ar-H); MS: m/z $211(\mathrm{M}+1)$. Anal. Cal. for $\mathrm{C}_{14} \mathrm{H}_{10} \mathrm{O}_{2}$ (210): C, 79.98; H, 4.79; Found: C, 79.94; H, 4.76\%.

\section{Single X-ray crystallography data collection, solution,} refinement, and structure elucidation

In compound 6 (Figure 1), all bond lengths were within normal ranges and comparable to those observed in the related structures. The three-ring system was not planar. The dihedral angle between the two benzene rings was $4.7(1)^{\circ}$. $\pi-\pi$ Interactions with distances $C g 1 \ldots . . .62^{\mathrm{i}}=3.605$ (1) $\AA$ (symmetry code: $1-x,-y,-z) ; C g 2 \ldots . . C g 2^{\mathrm{i}}=3.850$ (1) $\AA$ and $C g 3 \ldots . . C g 1^{\mathrm{ii}}=3.580$ (1) A [symmetry codes: (i) $1-x,-y,-z$; (ii) $2-x,-y, 1-z], C g 1, C g 2$ and $C g 3$ are the centroids of C9/C14/C11-C13, C1-C4/C11/C14 and $\mathrm{C} 5-\mathrm{C} 8 / \mathrm{C} 13 / \mathrm{C} 12$ rings, respectively, from stacks of the molecules propagated. All $\mathrm{H}$ atoms were positioned geometrically and were treated as riding on their parent $\mathrm{C}$ atoms, with $\mathrm{C}-\mathrm{H}$ distances of $0.93-0.96 \mathrm{~A}^{\circ}$; and with $U_{\text {iso }}(\mathrm{H})=1.2-1.5 U_{\mathrm{eq}}$ (C).

\section{Data collection:}

Oxford Diffraction Xcalibur Sapphire3 diffractometer, Radiation source: fine-focus sealed tube Graphite monochromator $\omega$ scans, Absorption correction: multi-scan (CrysAlis PRO; Oxford Diffraction, 2010) $T_{\min }=0.890, T_{\max }=1.000,10601$ measured reflections, 2028 independent reflections, 1262 reflections with $I>$ $2 \sigma(I), R_{\text {int }}=0.033, \vartheta_{\max }=26.0^{\circ}, \vartheta_{\min }=3.6^{\circ}, h=-10 \rightarrow 10$, $k=-10 \rightarrow 10, l=-10 \rightarrow 10$. 


\section{Crystal data:}

$\mathrm{C}_{14} \mathrm{H}_{10} \mathrm{O}_{2}, \mathrm{Mr}=210.22$, Triclinic, $P 1$, Hall symbol: $-\mathrm{P} 1, a=8.2678$

(7) A, $b=8.5268$ (6) A, $c=8.5965$ (7) A, $\alpha=92.650(6)^{\circ}, b=116.592$

$(8)^{\circ}, y=104.045(7)^{\circ}, V=517.28(7) \mathrm{A} 3, Z=2, F(000)=220, D x=$

$1.350 \mathrm{Mg} \mathrm{m}^{-3}$, Mo $K \alpha$ radiation, $\lambda=0.71073 \mathrm{~A}^{\circ}$, Cell parameters from 3639, reflections, $\vartheta=3.6-29.1^{\circ}, \mu=0.09 \mathrm{~mm}^{-1}, \mathrm{~T}=293 \mathrm{~K}$, Block, white, $0.30 \times 0.20 \times 0.20 \mathrm{~mm}$.

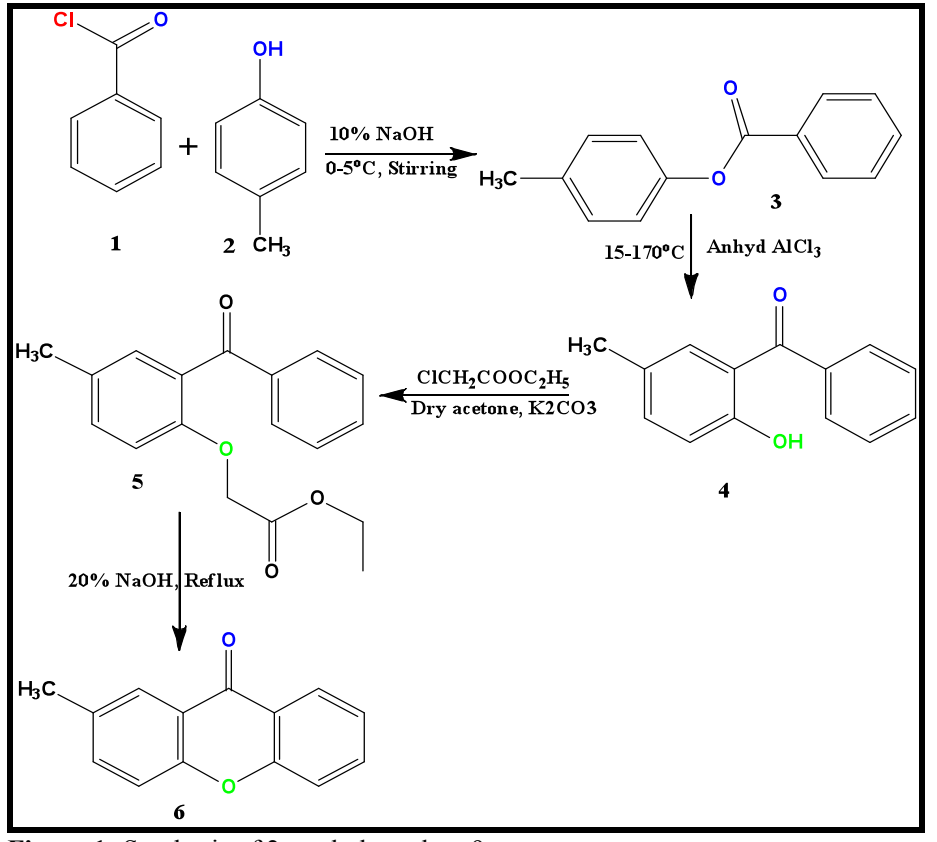

Figure 1: Synthesis of 2-methyl xanthen-9-one.

\section{Refinement:}

Refinement on $F^{2}$, Least-squares matrix: full, $R\left[F^{2}>2 \sigma\left(F^{2}\right)\right]=$ $0.052, w R(F 2)=0.152, S=1.04,2028$ reflections, 146 parameters, 0 restraints, Primary atom site location: structure-invariant, direct methods Secondary atom site location: difference Fourier Map, Hydrogen site location: inferred from neighboring sites, $\mathrm{H}$-atom parameters constrained, $w=1 /\left[\sigma^{2}\left(F_{\mathrm{O}^{2}}\right)+(0.0652 P)^{2}+0.0822 P\right]$, where $P=\left(F_{\mathrm{o}^{2}}+2 F_{\mathrm{c}^{2}}\right) / 3,(\Delta / \sigma) \max <0.001, \Delta \rho \max =0.13 \mathrm{e} \mathrm{A}^{-3}$, $\Delta \rho \min =-0.15$ e A $^{-3}$

The optimized structure of the title molecule is depicted in Fig 1(b) showing good agreement with the experimental results. This indicates the accuracy of B3LYP functional to predict the geometries of the organic compounds

\section{In vitro antimicrobial activity:}

The microbial strains were procured from the National Chemical Laboratory (NCL), Pune, India, and sub-cultured in our laboratory at optimum condition. The synthesized compound 6 $(50 \mu \mathrm{g} / \mathrm{ml})$ was screened against the following Gram-positive (Streptococcus pyogenes, Staphylococcus aureus, Bacillus subtilis), Gram-negative (Salmonella typhimurium, Klebsiella pneumonia, Escherichia coli) and fungal strains (Candida albicans, Aspergillus niger and Aspergillus flavus). The zone of inhibition is determined by disc diffusion method [16] and MIC values were determined by broth dilution method, using ampicillin in case of bacterial, ketoconazole for fungal as standard control and the results are presented in Table 1 and Table 2.

\section{Docking studies:}

The molecular interaction between protein and ligand can virtually be studied by subjecting the input files for molecular docking study. The docking operations can be performed by several algorithms, of which SURFLEX DOCK being such a program as available with SYBYL-X 2.1.1 software package (Tripos Inc. USA). The algorithm can readily be applied to rigid to flexible type docking. The input files as PDB file of protein and virtually sketched files of synthesized compounds were either collected from online server or drawn by using Chemdraw 15.0., and all other necessary calculations were performed as per default protocol [17]. Preamble to docking protocol, all the required input files were prepared, hydrogen atoms were added, water was removed, ionization state of C-terminal and $\mathrm{N}$ terminal were fixed and finally energy minimized by steepest descent method applying Gasteiger-Marsili charges and MMFF94s forcefield to the protein file with 100 iterations of conjugate gradient method with $1.0 \mathrm{kcal} / \mathrm{mol}$ as the convergence criteria fixing to 0.5 dielectric constant, whereas, ligand files were converted to 3D forms and energy minimized by applying Gasteiger-Huckel charges. The Geom mode of Surflex-dock allows the ligand files to flexibly interact with rigid protein files. The binding site of the protein file was abstracted from co-crystal ligand bound information and protomol generation program uses such information to find suitable cavity for docking. The adopted docking protocol was validated using a comparison of the binding modes of co-crystalized ligand of the target protein FtsZ (PDB: $3 \mathrm{VOB})$ before and after the docking study. Highlighting feature of Geom mode is generation of 20 conformers for each ligand, which interacts to the protein individually in order to identify the most stable conformer having best binding pose and binding energy. The respective ligand poses and docking scores in terms of Total score, Crash score and Polar score were obtained and represented accordingly.

Density functional theory calculations (DFT):

The whole DFT calculations [18] were performed with B3LYP functional $[19,20]$ under 6-31G $(d, p)$ basis set by using Gaussian 09 [21] and Gauss View 0.5 programs [22]. DFT studies covered geometry optimization, Frontier molecular orbitals (FMOs): HOMO-LUMO energies and molecular electrostatic potential (MEP).

\section{Hirshfeld surfaces analysis and fingerprint plots:}

The intermolecular interactions of the title molecule are deeply investigated by Hirshfeld surface analysis approach by using Crystal Explorer 17.5 program [23]. The normalization of contact distance $\left(d_{\text {norm }}\right)$ (see eqn. (1)) permits us to investigate the intermolecular interactions regions, where $d_{i}$ and $d_{e}$ are the distance from the Hirshfeld surface to the nearest atom inside and outside the surface, respectively. The combination of $d_{e}$ and $d_{i}$ in the form of 2D fingerprint plot enables us to summaries the intermolecular contacts in the crystal lattice

$d_{\text {norm }}=\left(d_{i}-r_{i}^{\mathrm{vdw}} / r_{i}^{\mathrm{vdw}}\right)+\left(d_{e}-r_{e}^{\mathrm{vdw}} / r_{e}^{\mathrm{vdw}}\right)$

where $\mathrm{r}_{i}^{\mathrm{vdW}}$ and $\mathrm{r}_{e}^{\mathrm{vdW}}$ being the van der Waals radii of the atoms. 


\section{BIOINFORMATION}

\section{Discovery at the interface of physical and biological sciences}

a

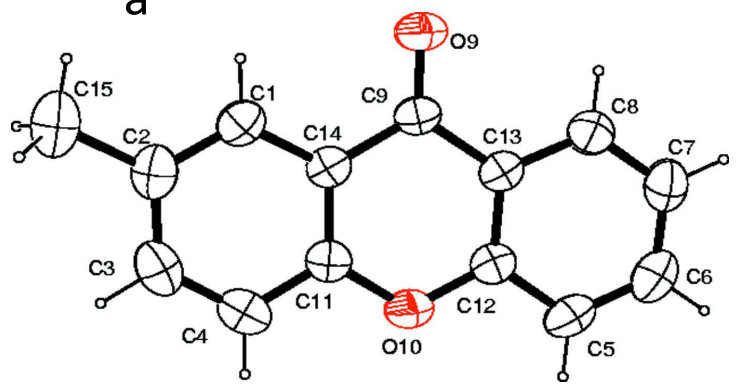

b

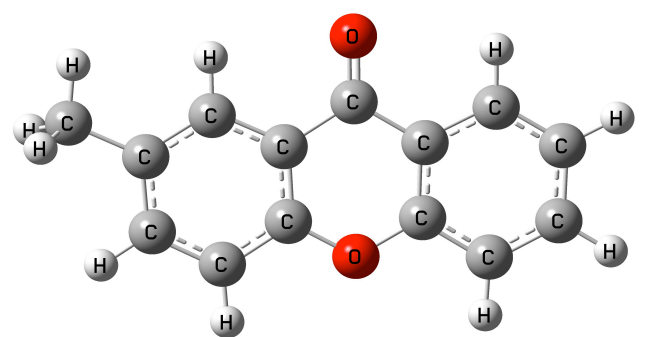

Figure 2. Structure of the title molecule: a) ORTEP with the atom-labelling scheme. The displacement ellipsoids are drawn at the $40 \%$ probability level. $\mathrm{H}$ atoms are shown as small spheres of arbitrary radii, and b) optimized structure at DFT/B3LYP/6-31G(d,p) level.

A)

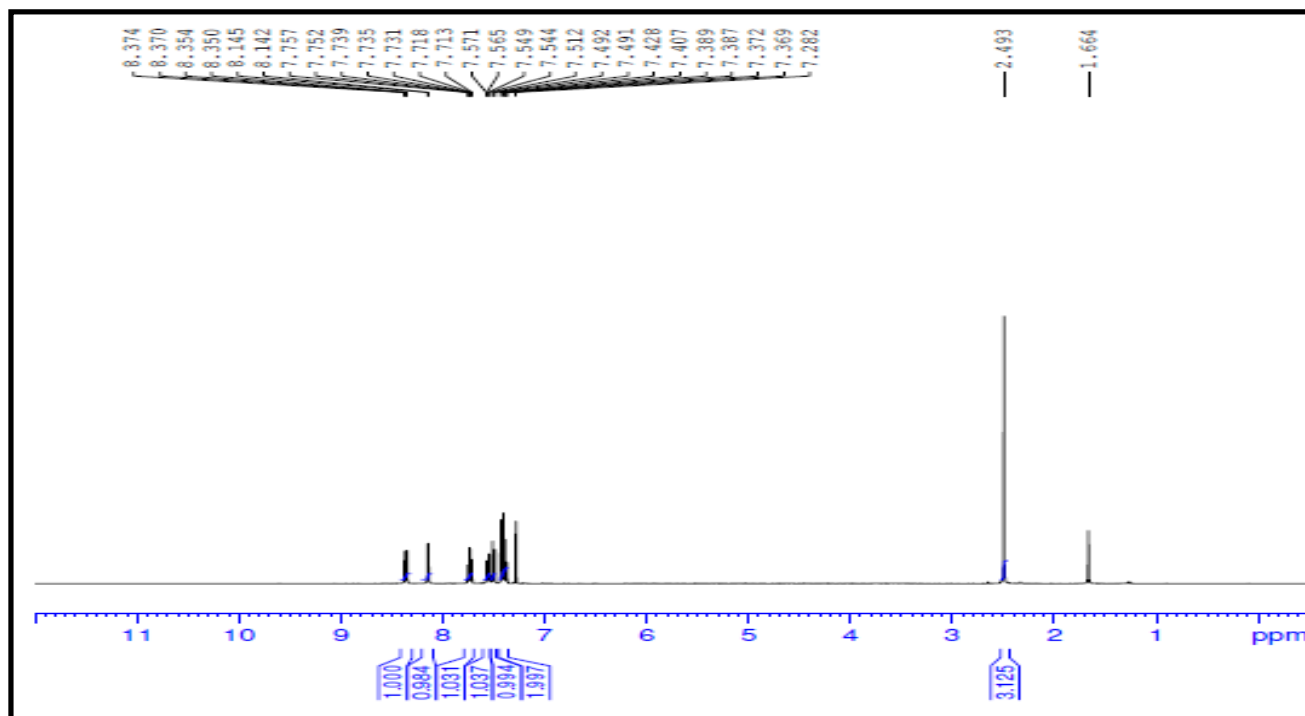

B)

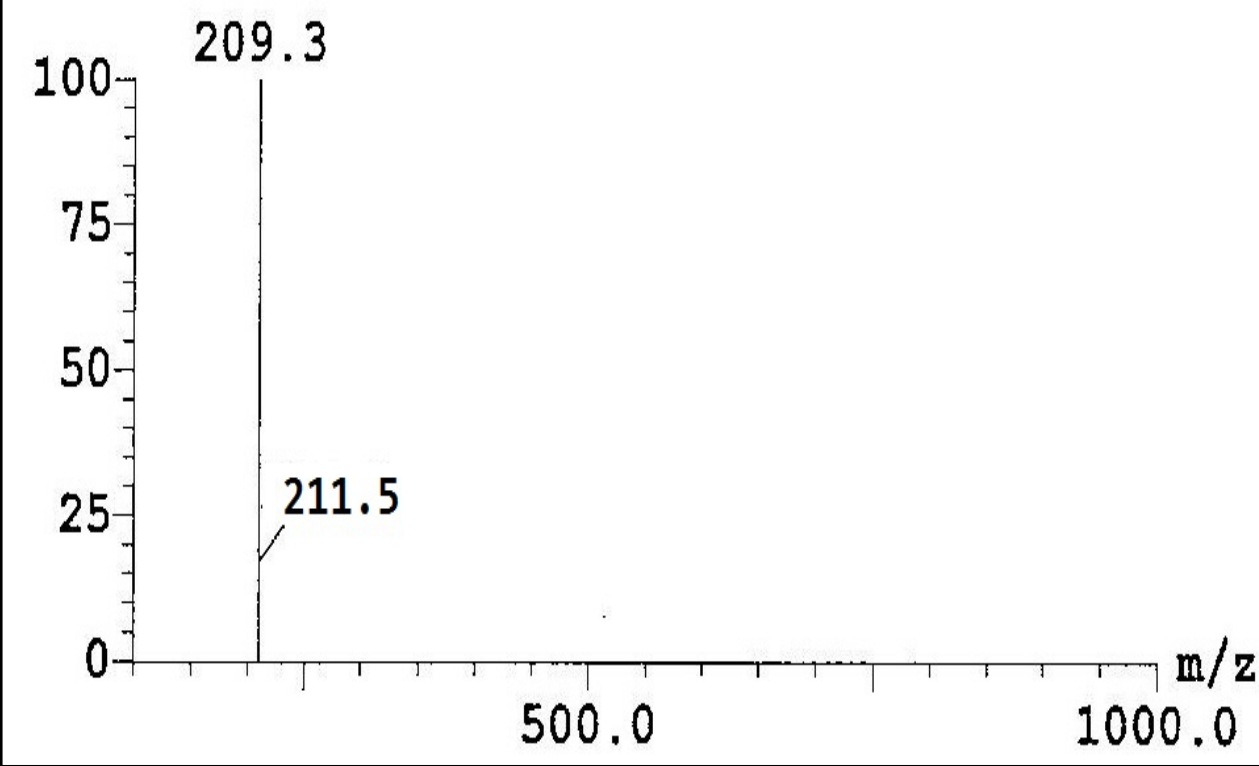

Figure 3: ${ }^{1} \mathrm{HNMR}$ and Mass Spectrum of compound 6. 
Table 1: Zone of inhibition of compound 6 against different bacteria and fungi at $1000 \mathrm{~g} / \mathrm{mL}$ concentration.

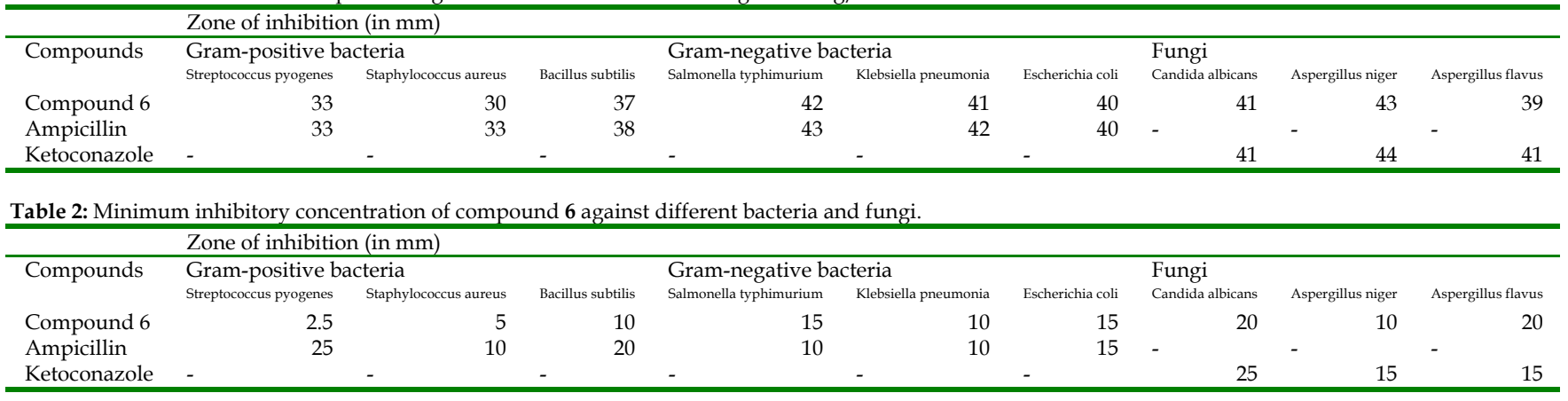

\section{Results and Discussion:}

The structure of the newly synthesized compounds was established on the basis of ${ }^{1} \mathrm{H}$ NMR, IR, mass, elemental analysis and X-ray diffraction studies. In IR spectra of benzoylated product (3), the emergence of $\mathrm{O}-\mathrm{C}$ stretching band for ester group and in proton NMR the disappearance of broad singlet for $\mathrm{OH}$ proton of p-cresol (1) indicates the formation of compound (3). Further, fries' rearrangement of compound (3) gave 2-hydroxy benzophenone (4), which was confirmed by the disappearance of $\mathrm{O}-\mathrm{C}$ stretching band and appearance of $\mathrm{O}-\mathrm{H}$ stretching band in IR spectra. In addition, the appearance of broad singlet for $\mathrm{OH}$ proton and decrease in one aromatic proton in proton NMR also confirmed the product formation. Compound 4 on etherification with chloro ethylacetate gave substituted ethyl ester (5), which is confirmed by the disappearance of $\mathrm{O}-\mathrm{H}$ stretching band and appearance of O-C stretching band for ester group in IR absorption spectra. Confirmation was also by observing the disappearance of broad singlet for $\mathrm{OH}$ proton and appearance of triplet and quartet for $\mathrm{CH}_{3}$, and $\mathrm{CH}_{2}$ protons respectively in proton NMR. Finally, the synthesis of compound 6 was confirmed by the appearance of $\mathrm{C}-\mathrm{O}-\mathrm{C}$ stretching in IR spectra and disappearance of singlet, triplet and quartet for $\mathrm{CH}_{2}$ and $\mathrm{CH}_{2}$ $\mathrm{CH}_{3}$ protons and decrease in one aromatic proton in ${ }^{1} \mathrm{HNMR}$ spectrum (Figure 2) is highly evidenced. Further, in mass spectrum the $\mathrm{m}^{+}$peak at 211.5 was exhibited in positive mode (Figure 2). In addition, the compound 6 was confirmed by X-ray crystal diffraction studies (Figure 1).

After confirmation of the newly prepared compound 6, they were tested against a panel of Gram-positive and Gram-negative bacteria as well as a few strains of fungi (Tables 1 and 2) using ampicillin and ketoconazole as standards for bacteria and fungi, respectively. Compound $\mathbf{6}$ showed excellent antimicrobial activity against all test microorganisms. These positive results may be due to the presence of a fused three ring system with -
$\mathrm{CH}_{3}$ and $\mathrm{C}=\mathrm{O}$ groups in the new molecule and are in agreement with several previous studies [24] Because of the carbonyl group in compound 6 similar to that present in ampicillin and ketoconazole molecule, the effect could be comparable with that of the standard drug.

Table 3: HOMO-LUMO and global chemical parameter values of the title molecule.

\begin{tabular}{lll}
\hline Property & Chemical parameters & Value \\
\hline HOMO energy & $\mathrm{E}_{\mathrm{H}}(\mathrm{eV})$ & -6.275 \\
LUMO energy & $\mathrm{E}_{\mathrm{L}}(\mathrm{eV})$ & -1.880 \\
Energy gap & $\Delta \mathrm{E}_{\mathrm{gap}}=\mathrm{E}_{\mathrm{L}}-\mathrm{E}_{\mathrm{H}}(\mathrm{eV})$ & 4.395 \\
hardness & $\eta=\left(\mathrm{E}_{\mathrm{L}}-\mathrm{E}_{\mathrm{H}}\right) / 2$ & 2.198 \\
Softness & $\sigma=1 / \eta$ & 0.455 \\
Chemical potential & $\mu=\left(\mathrm{E}_{\mathrm{L}}+\mathrm{E}_{\mathrm{H}}\right) / 2$ & 4.078 \\
Electronegativity & $\chi=-\sigma$ & -4.078 \\
Electrophilicity & $\omega=\mu^{2} / 2 \tau$ & 3.783 \\
\hline
\end{tabular}

A molecular docking study fundamentally defines the binding modes of ligand interaction at the active site of the receptor [17]. In our study the active Xanthone compound with notable antibacterial activity was subjected for docking studies against FtsZ protein and the crash score (revealing the inappropriate penetration into the binding site), Polar score (reports the polar region of the ligands) and total score was found to be -1.0241, 0.2314 and 5.6787 respectively. The inhibition of FtsZ (filamentous temperature sensitive protein $Z$ ) protein prevents the formation of divisome and hence it is a striking target for antibiotic research [25]. The binding interactions with the compounds revealed that compound finds well in the active pocket of the protein. The carbonyl oxygen of the chromone involves in p-donor hydrogen bonding with GLY196. The fused phenyl ring forms p-amide interaction with ASP199. The other phenyl ring involves in p-donor hydrogen and p-alkyl interaction with THR309 and LEU200 amino acid respectively. These interactions make the molecule to bind with protein molecule and hence responsible for showing desired activity (Figure 3). 


\section{BIOINFORMATION \\ Discovery at the interf face of physical and biological sciences}

A)

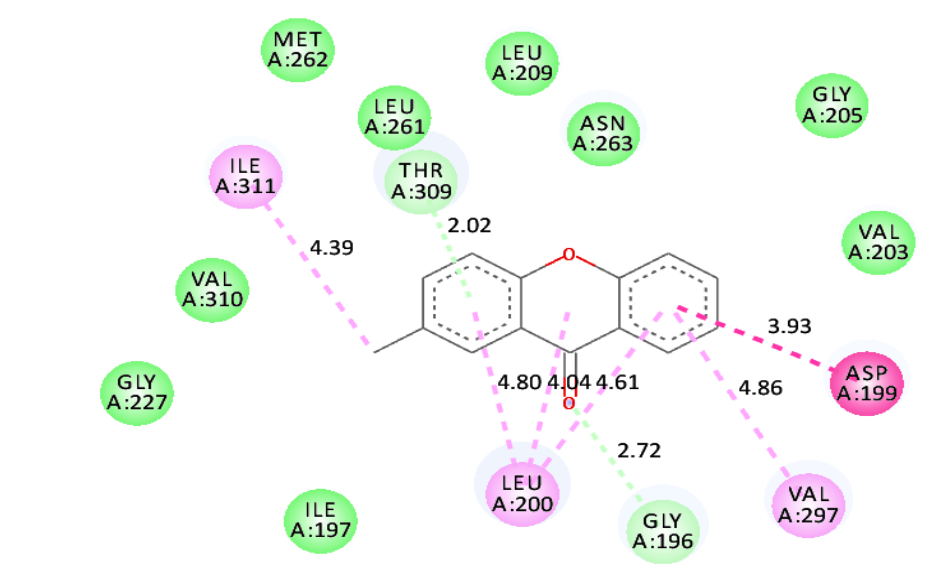

B)
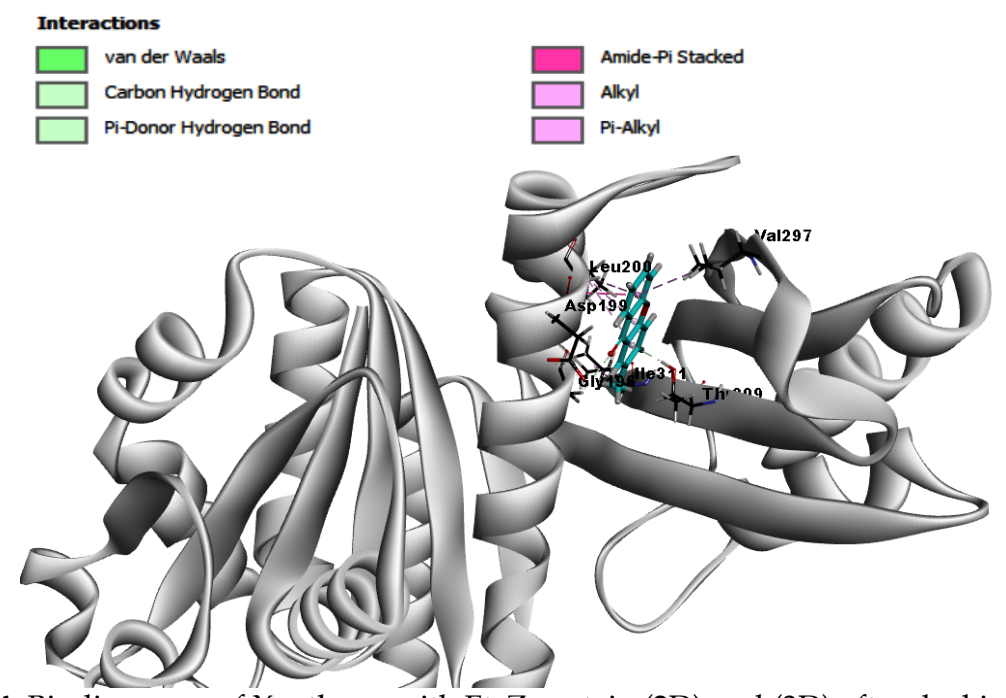

Figure 4: Binding pose of Xanthone with FtsZ protein (2D) and (3D) after docking studies

The optimized structure of the title molecule using density functional theory with B3LYP functional under 6-31G $(\mathrm{d}, \mathrm{p})$ basis set is illustrated in Figure 1 (b). The comparison of optimized structure with the experimental results showed good agreement. This indicates the accuracy of B3LYP functional to predict the geometries of the organic compounds. The optimized structure has been taken as an input to predict the others DFT calculations as described below: The stability of the compounds is mainly influenced by FMOs (i.e., HOMO and LUMO), which donates the highest occupied and lowest unoccupied molecular orbitals, respectively [26]. The energy difference between HOMO and LUMO is referred as energy gap $\left(\Delta \mathrm{E}_{\text {gap }}\right)$. It plays a critical role in determining the molecular electrical transport properties and enables us to determine the kinetic stability, chemical reactivity, optical polarizability and chemical hardness-softness of a molecule [27]. Mostly small $\Delta \mathrm{E}_{\text {gap }}$ lead to the ease of transporting electrons from HOMO level to LUMO level. The surfaces of HOMO (ground state) and LUMO (1 ${ }^{\text {st }}$ excited state) are sketched in Figure 4. The HOMO orbitals exhibited the predominant of $\pi$ character of electron density on the entire skeleton of molecule exception of the oxygen atoms that showed pure $\sigma$ character of electron density distribution. The highly concentration of $\pi$ electron density over the rings resulted in multiple stacking interactions of title compound with different amino acids as shown in Figure 3, indicating the agreement between DFT and docking studies. On the other side, the electron density distribution over LUMO orbital showed mix of $\sigma$ and $\pi$ character, but methyl group did not exhibit any type of electron density distribution. The HOMO and LUMO energies are found to be 6.275 and $-1.880 \mathrm{eV}$, respectively. Hence, the calculated energy gap is $4.395 \mathrm{eV}$. The lower energy gap explains the charge transfer interactions taking place within the molecule, which recently, used to prove the bioactivity from intramolecular charge transfer [28]. Table 3 lists the energies of HOMO, LUMO, $\Delta \mathrm{E}_{\text {gap }}$ as well as other global reactivity parameters. 

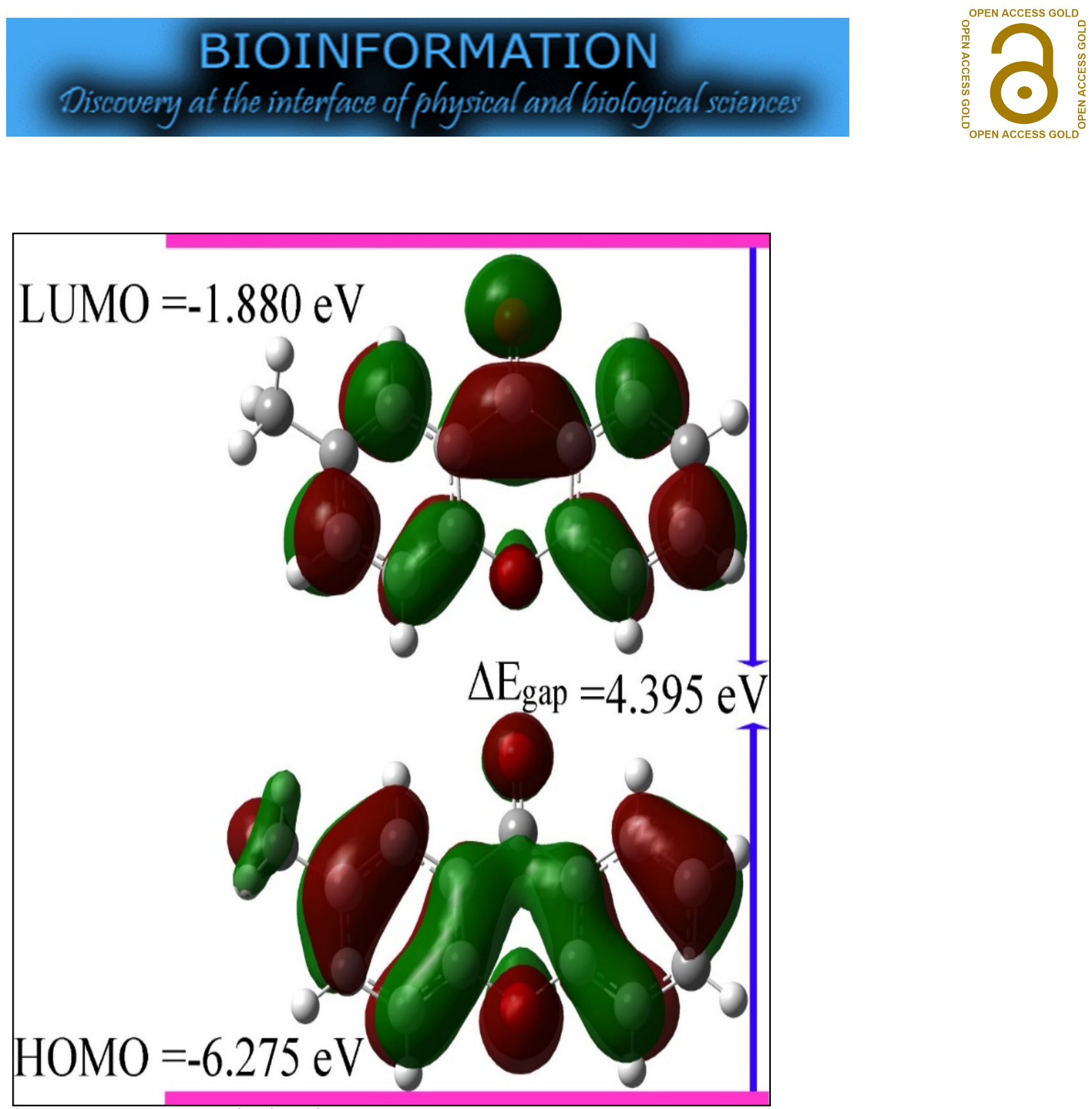

Figure 5. HOMO-LUMO orbitals with energy gap

The mapping of MEP is a representation of the electrostatic potential mapped upon is surface of electron density showing the 3D charge distributions of a molecule [29]. This information about the charge distributions enables us to predict how the molecule can interact with others. Figure 5 represents MEP of the title molecule with colours scaled from -7.166 e-2 (deepest red) to 7.166 e-2 (deepest blue), whereas the intermediary colours indicating the intermediary electrostatic potentials. The maximum negative electrostatic potential (electronegativity) appears around the oxygen atom of the carbonyl oxygen (O9), which indicate the nucleophilic site reaction of the molecule. This result is agreed with docking studies that showed formation of hydrogen bond between O9 and GLY196 residue (see Figure 3, Molecular docking studies). On the other hand, the maximum positive electrostatic potential appears around the hydrogen atoms of the phenyl ring especially $\mathrm{H} 3$ and $\mathrm{H} 7$ atoms, which indicate the electrophilic site reaction of the title molecule.

The Hirshfeld surface of the title molecule was mapped over volume of $253.15 \AA^{3}$ and area of $246.12 \AA^{2}$, respectively. Figure 6 present Hirshfeld surface over $d_{\text {norm }}$ with colour range from -0.0185 a.u. (red) to 1.2012 a.u. (blue) represent the distance shorter and greater the sum of vdw radii of the contacted atoms, respectively. The white color represents the distance equal to the sum of vdw radii. The dark red spots on the surface donate the hydrogen bonds. The fingerprint plots of the title molecule for all atoms are depicted in figure 7(a). Furthermore, the decomposition of fingerprint plots of a particular pair of close contact atoms are depicted in figure $7(\mathrm{~b}-\mathrm{d})$. H...H inter contact (Fig. 7(b)) showed as a huge wing pointing at $d_{e}=d_{i} \approx 1.18 \AA$ has an influence to the crystal packing with $49.1 \%$ contribution to the 

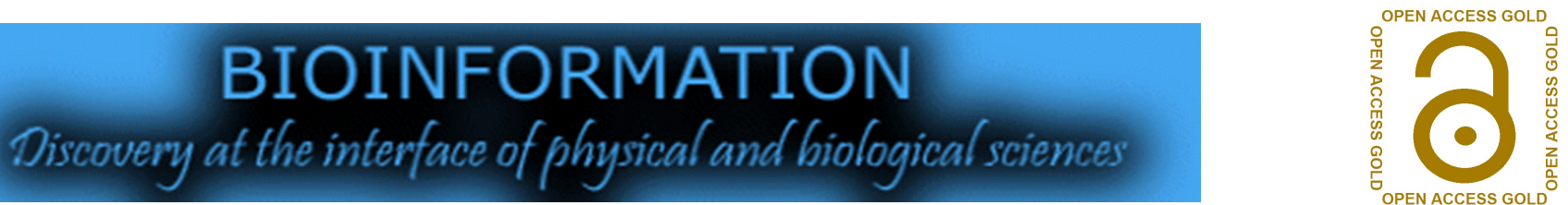

total Hirshfeld surface. While the $\mathrm{H}$...O/O...H intermolecular interaction (Figure 7) comprising only 19.2\% of Hirshfeld surfaces and appear as two characteristic wings have the major contribution toward crystal packing where the atoms contacted at distance less than the sum of vdw radii (i.e., $d_{e}+d_{i} \approx 2.66 \AA$ ). H..C/C ... H intermolecular interaction coated $15.5 \%$ of the Hirshfeld surface without significant contribution to the crystal packing of the title molecule. The contribution from C...C contacts $(13.8 \%)$ (Figure 7 ) confirms the presence of $\pi . . . \pi$ stacking in the crystal packing formation. This also could be shown on the shape index surface as red and blue triangles (Figure 6II) and also, as flat regions upon the curvedness surface (Figure 6 III).

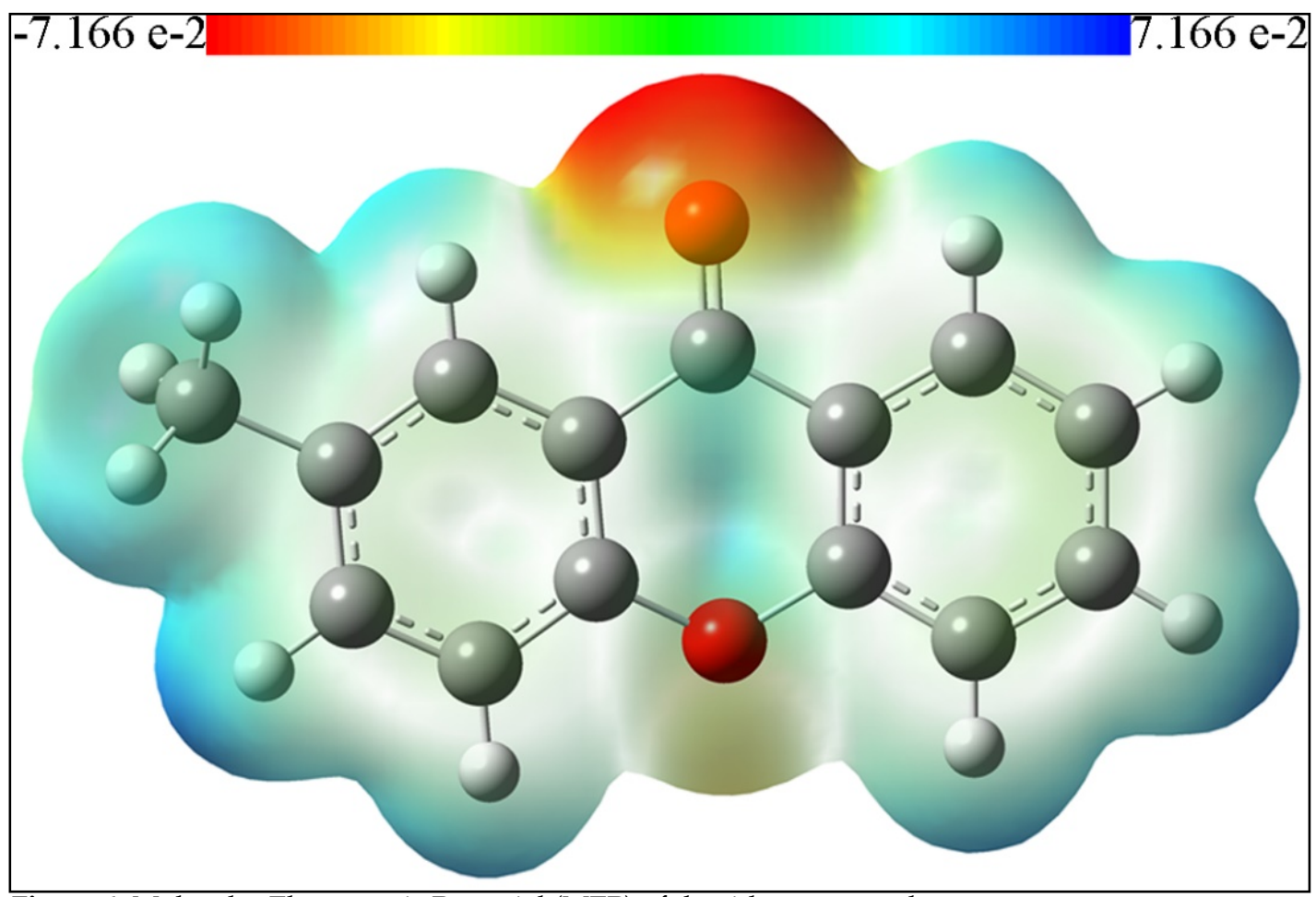

Figure 6. Molecular Electrostatic Potential (MEP) of the title compound

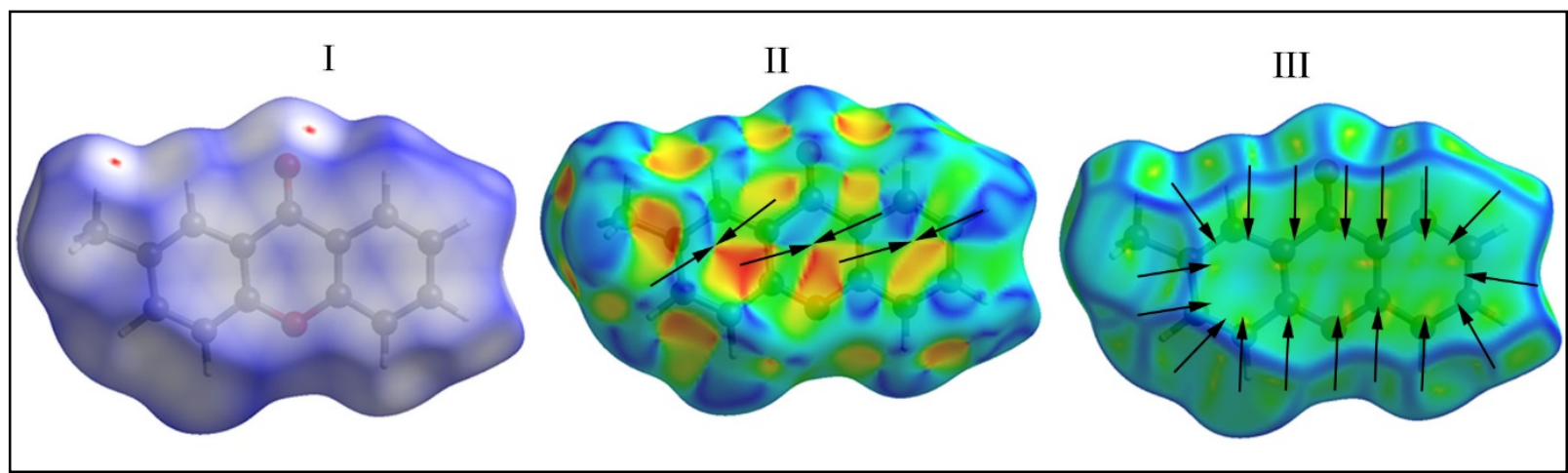

Figure 7. Hirshfeld surface with (I) $d_{\text {norm }}$ (II) Shape index and (III) Curvedness of the title molecule 


\section{BIOINFORMATION}

Discovery at the interf face of physical and biological sciences

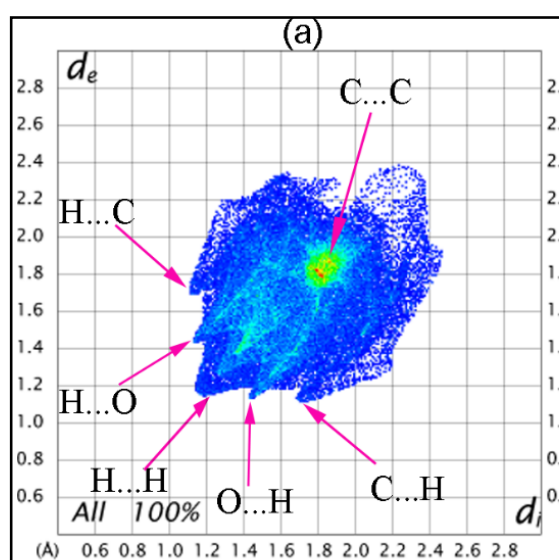

(c)

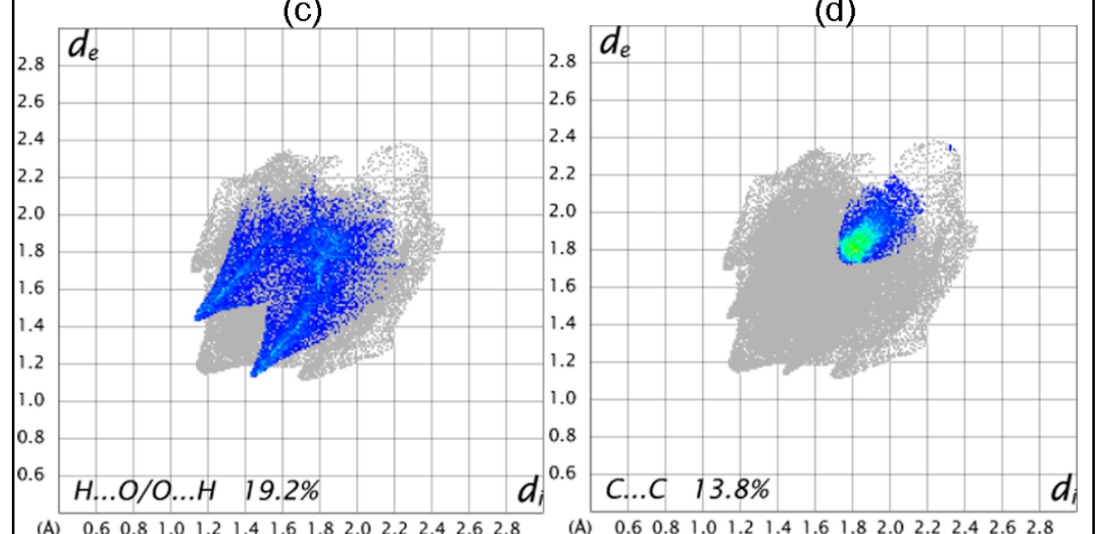

Figure 8. 2D fingerprint of the title compound: a) Overall contacts and (b-d) represent decomposition of the contacts of particular pairs of atoms.

\section{Conclusion:}

We document the design, synthesis, docking, Hirshfeld surface analysis and DFT calculations of 2-methylxanthen-9-with the FtsZ protein (PDB ID: 3VOB) from Staphylococcus aureus for antimicrobial applications.

\section{Conflict of interest:}

No conflicts of interest associated with this work.

\section{Acknowledgment:}

We the authors express our sincere gratitude to the Principal, The National Institute of Engineering, Mysuru, JSS College of Arts, Commerce and Science, Ooty Road, Mysuru and JSS Academy of Higher Education and Research, Mysuru for encouragement and support to carry out the research work.

\section{References:}

[1] Govindachari TR et al. Tetrahedron 1971 27:3919.

[2] De Oliveira WG et al. Phytochemistry, 1972; 11:3323.

[3] Chaivisuthangkura A et al. Chromatographia, 2009 69:315.

[4] Aqil F et al. Journal of Basic Microbiology 2005 45:106.

[5] Shankaranarayan D et al. Archives Internationales de Pharmacodynamie et de Therapie, 1979 239:257.

[6] Ho CK et al. Planta Medica, 2002 68:975.

[7] Chen SL et al. Journal of Liquid Chromatography and Related
Technologies, 2005 28:2953.

[8] Lai CK. Thesis Titled: Synthesis and Characterisation of 1, 3Dihydroxyxanthone Derivatives and Their Antioxidant Activities, Universiti Tunku Abdul Rahman, Malaysia, 2012.

[9] Teixeira M et al. Pharmaceutical Development and Technology 2019 24:1104.

[10] Cidade H et al. Arabian Journal of Chemistry, 2020 13:17.

[11] Alam S \& Khan F Scientific Reports, 2018 8:1.

[12] Fernandes C et al.Molecules, 2019 24:791.

[13] Araujo et al. Molecules, 2019 24:314.

[14] Avin BRV et al. European Journal of Medicinal Chemistry, 2014 75:211.

[15] Prashanth T et al. Structure Reports Online, 2012 68:o2435.

[16] Shirahatti et al. 2020

[17] Mandal et al. Chemistry Central Journal, 2018 12, p.141.

[18] Hohenberg P \& Kohn W, Inhomogeneous electron gas, Phys. Rev. 1964 136:B864.

[19] Becke AD, J. Chem. Phys. 1993 98:5648.

[20] Lee C et al. Phys. Rev. B 1988 37:785

[22] Dennington R et al. GaussView 5.0, Semichem Inc., Wallingford. 2009

[23] Turner $\mathrm{M}$ et al. Book Manual for CrystalExplorer. Version 17 software, University of Western Australia. Australia, 2017.

[24] Padhi S Phytochemistry, 2019; 157:175-83.
[21] Frisch MJ et al., Gaussian 09, Gaussian Inc, Wallingford, 2009. 


\section{BIOINFORMATION}

\section{Discovery at the interf ace of physical and biological sciences}

[25] Sangeeta GPV Microbial pathogenesis, 2018 124, pp.258-265.

[26] Fleming I, Frontier orbitals and organic chemical reactions; Wiley \& Sons. USA, 1977.
[27] Jensen F, Introduction to Computational Chemistry, Pp 522, Ch 16 Qualitative Theories, 16.3 Qualitative Molecular R Molecular Orbital Theory, John Wiley \& sons, 2017.

[28] Khamees HA et al. J. Mol. Struct. 2018 1161:199.

[29] Okur M et al. J. Phys. 2018 48:398.

Edited by P Kangueane provided the original work is properly credited. This is distributed under the terms of the Creative Commons Attribution License

\section{Articles published in BIOINFORMATION are open for relevant post publication comments and criticisms, which will be published immediately linking to the original article for FREE of cost without open access charges. Comments should be concise, coherent and critical in less than 1000 words.}




\section{BIOINFORMATION}

\section{Discovery at the interface of physical and biological sciences}
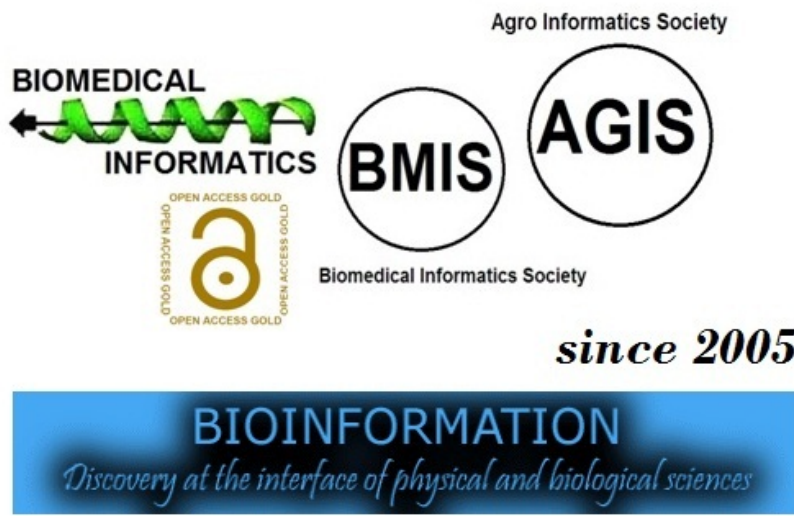

indexed in
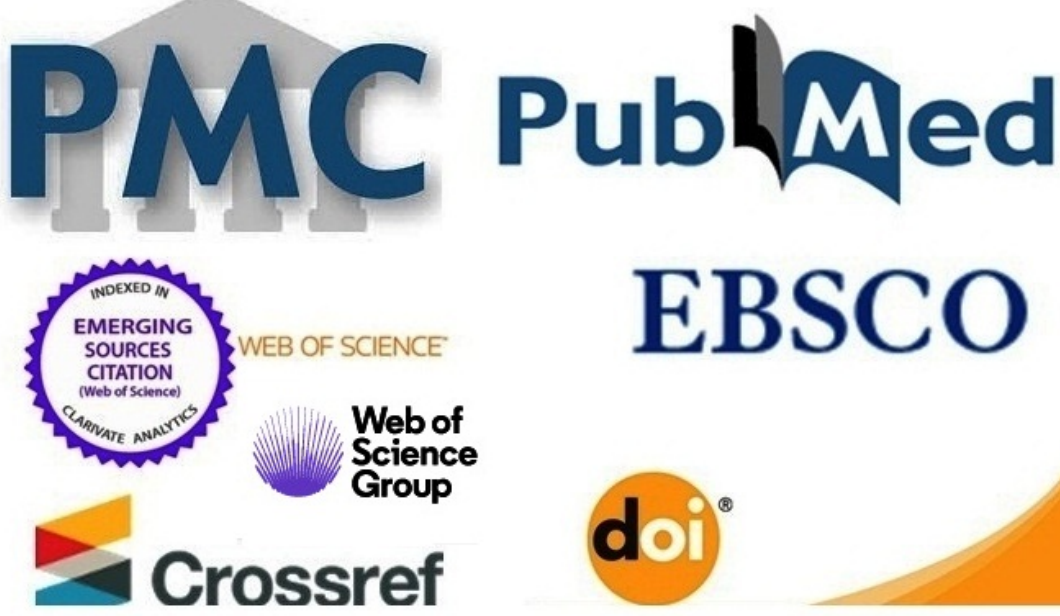

Neb of

Science

Group
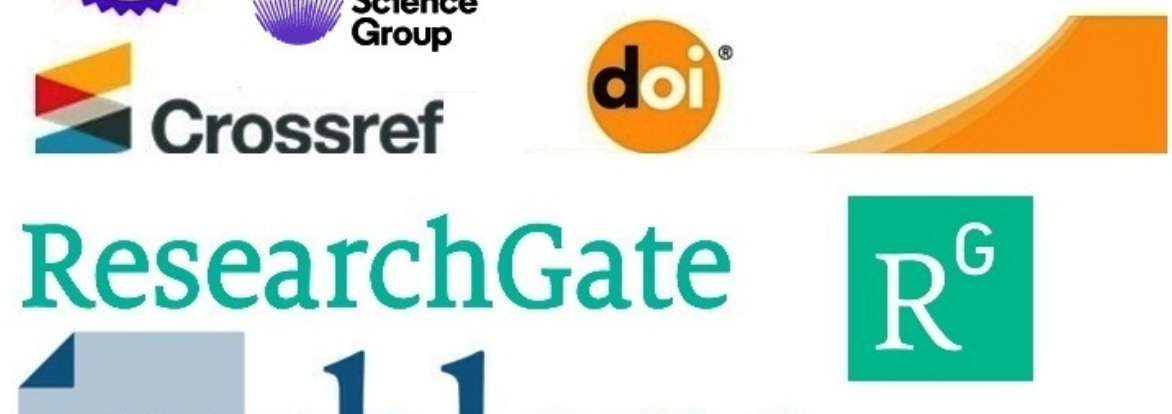

publons 